\title{
CORRIGENDUM
}

\section{The estimation of the nutritive value of feeds as energy sources for ruminants and the derivation of feeding systems}

K. L. BLAXTER AND A. W. BOYNE

J. agric. Sci., Camb. (1978), 90, 47-68

The authors regret the following error:

On p. 53, equation (11) is incorrect and should read:

$$
E_{M, f}=\frac{E_{G, f}}{q^{*}}=\frac{B-1}{B G^{*} q^{*}}
$$

